\title{
SecurAcath for Securing Peripherally Inserted Central Catheters: A NICE Medical Technology Guidance
}

\author{
Tom Macmillan $^{1}$ (D) Mark Pennington ${ }^{2} \cdot$ Jennifer A. Summers ${ }^{3} \cdot$ Kate Goddard $^{1} \cdot$ Darshan Zala $^{2} \cdot$ Naomi Herz $^{1}$. \\ Janet L. Peacock ${ }^{3}$. Stephen Keevil ${ }^{1}$. Anastasia Chalkidou ${ }^{1}$
}

Published online: 20 August 2018

(c) The Author(s) 2018

\begin{abstract}
Central venous catheters are commonly used to deliver therapies and to monitor patients, and require securing at the point of percutaneous entry to avoid dislodgement. SecurAcath is a catheter securement device designed for central venous catheters. The National Institute for Health and Care Excellence, as a part of its Medical Technologies Evaluation Programme, selected this device for evaluation and invited the manufacturer, Interrad Medical, to submit clinical and economic evidence. The King's Technology Evaluation Centre, an External Assessment Centre commissioned by the National Institute for Health and Care Excellence, independently critiqued the manufacturer's submissions. The External Assessment Centre found a lack of evidence comparing SecurAcath with alternative approaches to securement (StatLock, suturing, tape securement), with one unpublished randomised controlled trial providing the strongest evidence. The External Assessment Centre conducted a new systematic review and meta-analysis and concluded that there is some evidence indicating the non-inferiority of SecurAcath compared to StatLock. The External Assessment Centre considered the manufacturer's economic model to be appropriate but made revisions to some parameters and noted significant heterogeneity in the included studies. The revised model indicated that StatLock was more cost effective than SecurAcath for catheter indwell times of up to 5 days; however, for medium- and long-term indwell times, SecurAcath was the most cost-effective option. The National Institute for Health and Care Excellence Medical Technologies Guidance MTG 34, issued in June 2017, recommended the adoption of SecurAcath for securing peripherally inserted central catheters within the National Health Service in England.
\end{abstract}

Electronic supplementary material The online version of this article (https://doi.org/10.1007/s40258-018-0427-1) contains supplementary material, which is available to authorized users.

Tom Macmillan

thomas.macmillan@kcl.ac.uk

1 King's Technology Evaluation Centre, School of Biomedical Engineering and Imaging Sciences, King's College

London, 5th Floor, Becket House, 1 Lambeth Palace Road, London SE1 7EU, UK

2 King's Health Economics, Institute of Psychiatry, Psychology and Neuroscience, King's College London, London, UK

3 School of Population Health and Environmental Sciences, King's College London, London, UK

\section{Key Points for Decision Makers}

Available evidence suggests that SecurAcath is an effective catheter securement device and is easy to insert and maintain, well tolerated and associated with a low rate of catheter-related complications.

SecurAcath should be considered for any peripherally inserted central catheter with an anticipated indwell time of 15 days or longer.

SecurAcath is cost saving compared with adhesive securement devices, when the peripherally inserted central catheter is in places for 15 days or longer. Cost savings range from $£ 9$ to $£ 95$ per patient with a minimum annual saving of an estimated $£ 4.2$ million in the National Health Service in England. 


\section{Introduction}

The National Institute for Health and Care Excellence (NICE) promotes the adoption of clinically and cost-effective medical devices and diagnostics by the National Health Service (NHS) in England through the work of the Medical Technologies Evaluation Programme and Diagnostics Assessment Programme, which were established in 2009. Manufacturers of medical devices and diagnostics notify NICE when their product meets the eligibility criteria for entry to the programme. Technologies are selected for development of Medical Technologies Guidance by NICE's Medical Technologies Advisory Committee (MTAC) if they have the potential to offer a significant clinical benefit to patients and the NHS, at the same or reduced cost when compared with current practice. Once a technology has been selected, NICE prepares a scope outlining the population and outcomes for which the manufacturer should submit clinical and economic evidence. A NICE-funded External Assessment Centre (EAC) independently critiques the submitted evidence and prepares an assessment report. The EAC is also required to provide additional evidence if there are gaps in evidence submitted by the manufacturer. The MTAC uses the EAC report, together with other sources of advice, to produce guidance on the proposed technology [1, 2].

This article presents a summary of the EAC report for the SecurAcath catheter securement device for medium- and long-term central venous catheters and the development of the NICE guidance. The article is one among the series of NICE Medical Technology Guidance summaries published in Applied Health Economics and Health Policy [3].

\section{Background}

\subsection{Catheter Securement}

Long-term central venous catheters (CVCs) allow access to large blood vessels and are often used in chemotherapy where the drug can cause damage to the blood vessel if infused in smaller veins. Peripherally inserted central catheters (PICCs) are typically inserted in the arm (basilic or cephalic vein) with the tip of catheter placed in the superior vena cava, the right atrium of the heart or the inferior vena cava, located using ultrasound. Centrally inserted central catheters (CICCs) are inserted in the chest. Optimum tip position is in the lower third of the superior vena cava or the cavoatrial junction or within the inferior vena cava above the level of the diaphragm $[4,5]$.

Once in position, the catheter is secured at the entry site by specialised adhesive devices (such as StatLock), sutures, surgical tape or steri-strips, or by a subcutaneous securement device such as SecurAcath. The insertion site should be cleaned thoroughly on a weekly basis, during which dressings and any adhesive securement devices are removed and discarded. However, unless there is an indication of device malfunction or insertion-site infection, SecurAcath does not need to be removed at this stage.

Catheter removal before completion of the intended treatment is categorised as unplanned removal. This can occur as a result of migration, dislodgement, infection, phlebitis or thrombosis. In the case of catheter migration, the catheter may be repositioned or a new catheter may be put in place depending on how far the catheter has migrated: if a minor migration has occurred, the line can be salvaged. A malpositioned CVC is managed depending on the location of the CVC, the continued need for infusion therapy and the patient's acuity. Infusion through a malpositioned CVC should be withheld until a proper tip position has been established. A catheter that has migrated externally should not be re-advanced prior to re-stabilisation. If the CVC becomes dislodged, for example when the tip moves further out than the SCV, there is a higher risk of thromboembolism and appropriate management may require CVC exchange or removal and insertion at a new site [4]. Catheter-related blood stream infections (CRBSIs) often require catheter removal for effective treatment.

\subsection{National Institute for Health and Care Excellence Scope}

\subsubsection{Population}

Central venous catheters are used in a heterogeneous range of indications where venous access is required for either long- or short-term care. Indications for CVCs include the following (adapted from Smith and Nolan [6]).

- Access for drugs and nutrition

- Infusion of irritant drugs, for example, chemotherapy

- Total parenteral nutrition

- Poor peripheral access

- Long-term administration of drugs, such as antibiotics

- Access for extracorporeal blood circuits

- Renal replacement therapy

- Plasma exchange

- Extracorporeal membrane oxygenation

- Monitoring or interventions 
- Central venous pressure

- Central venous blood oxygen saturation

- Pulmonary artery pressure

- Temporary transvenous pacing

- Targeted temperature management

- Repeated blood sampling

Central venous catheters are also widely used in the treatment of chronic conditions, such as cancer. The National Audit Office suggested that 130,000 patients with cancer received chemotherapy in 2013-14 in all settings (hospital admissions, outpatient attendances and community care) [7] Typically, PICCs are used, but CICCs may be used in patients with poor vasculature or for certain types of chemotherapy. Patients with bowel diseases may also require $\mathrm{CVC}$ insertion owing to the nature of their disease and feeding requirements. Patient preference and tumour grading may also be considered when deciding on the vascular access device.

\subsubsection{Intervention}

SecurAcath (Interrad Medical Inc.) is a single-use securement device indicated for short- or long-term securement of percutaneous indwelling catheters for intravenous use by means of a subcutaneous anchor at the insertion site. It is not currently indicated for conventional peripheral intravenous catheters.

SecurAcath uses a small anchor that is placed just beneath the skin at the catheter insertion site. The anchor is attached to a base that is used to grip the catheter shaft to prevent inadvertent movement. The base is made up of two foldable metal legs and two securement feet. The feet are inserted under the skin at the catheter insertion site and are unfolded to form a subcutaneous anchor. SecurAcath is designed for round-shaft catheters.

SecurAcath was CE marked in February 2010, under Directive 93/42/EEC for Medical Devices.

The device is contraindicated whenever:

- Skin integrity is deemed unfavourable by the operator, for example, friable skin as a result of long-term corticosteroid use or the presence of cellulitis or rashes at the desired site of catheter insertion;

- Local tissue factors will prevent proper device stabilisation and/or access;

- The presence of device-related infection, bacteraemia or septicaemia is known or suspected;

- The patient's body size is insufficient to accommodate the size of the implanted device;

- The patient is known or is suspected to be allergic to materials contained in the device;
- The prospective insertion site has previously received irradiation.

There are six versions of SecurAcath. These are used with 3, 4, 5, 6, 7 and 8 French-size CVCs. All sizes have the same functionality. The anchor base and anchor sizes are the same for each version, only the channel diameter changes. A SecurAcath device must be selected to match the catheter diameter. If a catheter is labelled with a half French size, the closest smaller size SecurAcath should be used.

The device is used once a catheter has been placed following the standard procedure. The SecurAcath requires a minimum of $3 \mathrm{~cm}$ of catheter shaft exposed above the skin surface and the manufacturer recommends that a small incision to the skin of approximately $3 \mathrm{~mm}$ is made parallel to the shaft of the catheter.

To use the device, the anchor tips are inserted into the catheter insertion site. The anchor is then advanced as closely as possible to the catheter shaft and the anchor base is released. The anchor is then secured open by placing a cover over the anchor base. Once the device is secured, it lays flat on the skin. The site should then be dressed as per hospital protocol.

The device is removed at the same time that the catheter requires removal. This is done by removing the cover from the anchor base. The catheter is then removed. The anchor base can then be cut lengthways and each half removed separately or the anchor base can be folded and the anchors removed. Local anaesthesia may be used at the site prior to removal to reduce pain.

\subsubsection{Comparators}

Adhesive securement devices aim to increase attachment to the skin, thus minimising catheter movement and reducing complications. The StatLock adhesive securement device (Bard Access Systems) is commonly used within the NHS for catheter stabilisation. The StatLock is a single-use adhesive anchor device that uses a 'post and door' design, the doors locking the suture wings of the CVC to a foam-based anchor pad, which adheres firmly to the patient's skin and is removed using alcohol. The device must be changed at least every 7 days or along with dressing changes. The StatLock stabilisation device is compatible with medical tubes and catheters. The device is contraindicated in people with known tape or adhesive allergies or a known sensitivity to benzoin. Similarly, the Grip-Lok device (Zefon International) includes an adhesive base layer that attaches to the patient's skin. The catheter or catheter/connector combination is pressed onto an adhesive pad attached to an upper surface of the base layer. A Velcro closure layer is then folded over the catheter and attaches to the upper surface of the base layer. Central venous catheters may also be secured 
using surgical tape. Adhesive securement devices are most typically used with PICCs.

Sutures are surgical stiches used to hold together wounds or to secure devices. Sutures are typically used in short-term percutaneous (non-tunnelled) catheters and in skin-tunnelled catheters. They are not typically used in PICCs, but may be used in neonates or people likely to forcibly remove the line.

\subsubsection{Outcomes}

Central venous catheter complications can be related to insertion, indwelling or extraction and may be immediate (typically relating to insertion or extraction) or delayed (typically related to indwelling) [8]. Delayed complications associated with unsecured or poorly secured catheters include catheter migration or dislodgement, infection, thrombosis and phlebitis. The literature suggests CVC use may have complication rates of $1-26 \%$ [6].

The final scope stated that the following outcomes would be considered:

- Rates of migration and dislodgement;

- Rates of catheter-related infection (including CRBSI, local infection/inflammation and thrombophlebitis);

- Unplanned removals and reinsertions;

- Time taken to secure catheter;

- Patient and clinician satisfaction scores;

- Pain while in situ and on insertion and removal;

- Quality-of-life measures;

- Device-related adverse events, e.g. catheter malfunction, thrombosis and vessel erosion.

A table detailing the full final scope is included in the Supplementary Appendix.

\section{Review of Clinical and Economic Evidence}

The first part of the manufacturer's submission included clinical evidence, comprising an overview of the clinical indications and current treatment provisions, and a systematic review of clinical evidence related to SecurAcath and the comparators. The second part was a submission of the economic evidence comprising a systematic review of economic evidence and a de-novo economic model of SecurAcath and the comparators. The King's Technology Evaluation Centre, an EAC based at King's College London, was commissioned by NICE to critique the manufacturer's submission of clinical and economic evidence and to produce a structured assessment report. Nominated expert advisers were available to provide advice to the EAC during the preparation of the report.

\subsection{Clinical Effectiveness}

The manufacturer included 20 studies that reported on clinical outcomes, of which eight were excluded by the EAC. Four studies were narrative reviews and commentaries that were not considered relevant evidence, while another four studies were excluded because of methodological flaws.

Only one study directly compared SecurAcath with a competitor product (StatLock); this was a randomised controlled trial (RCT) focusing on the time taken to change a dressing [9]. Three studies evaluated SecurAcath without a comparator, [10-12] reporting catheter-related adverse events such as migration and dislodgement. Seven observational studies, published as poster presentations, compared SecurAcath with historical controls without explicitly describing the comparator [13-19].

The EAC critically appraised these studies and presented a narrative review of how they reported the clinical effectiveness of SecurAcath. Eleven studies were deemed to have 'low' relevance to the decision problem; six studies were deemed to have 'medium' relevance; and one study 'medium/high' relevance (the only RCT comparing SecurAcath to a comparator, StatLock) [9].

\subsubsection{Meta-Analysis}

The manufacturer concluded that there was not enough evidence to perform a meta-analysis. However, despite the limitations in the available evidence on SecurAcath (several studies are presented as conference abstracts or poster presentations), the EAC concluded it was possible to metaanalyse six of the outcomes considered in the original scope (see below). Studies containing overlapping populations, or where the SecurAcath was combined with another securement intervention (e.g. glue) were excluded from the meta-analysis.

The six outcomes considered for meta-analysis were:

- Migration;

- Dislodgement;

- Catheter-related infection;

- CRBSI;

- Unplanned removals;

- Unplanned reinsertions.

For SecurAcath, six studies included by the manufacturer were used in the meta-analysis [10-12, 15, 16, 19] along with two others identified by the EAC [20, 21]. Another eight studies that reported on the comparators (StatLock and suturing) $[9,22-28]$ were included by the EAC. Outcome data were extracted and the raw values calculated based on the available evidence. Relative risks and 95\% confidence intervals (CIs) were calculated for each required 
RCT outcome using the Cox-Hinckley-Miettinen-Nurminen method for small samples [29]. Pooled relative risk could not be calculated for non-comparative studies, thus the fixed-effects pooled proportions and 95\% CIs were calculated for all studies by intervention and outcome separately. There were too few studies to investigate publication bias. The EAC noted that many SecurAcath studies were not full peer-reviewed papers but were available only as poster presentations or conference abstracts, although there were more peer-reviewed published studies on StatLock.

\subsubsection{Results}

Relative risk calculations, based entirely on the single RCT for SecurAcath vs. StatLock, [9] are presented in Table 1. The estimates are imprecise owing to the relatively small sample sizes. Pooled estimates by intervention and outcome are presented in Table 2. With the exception of dislodgement, the $95 \%$ CIs for migration, total catheterrelated infections and CRBSIs were overlapping between SecurAcath and StatLock.

The majority of the observational studies reported higher pain scores during device removal in comparison with device placement and in situ. The most relevant study for UK practice that reported pain scores [10] stated that device removal caused the most dissatisfaction among staff and that patients were complaining of pain or discomfort. Although not included as an outcome in the meta-analysis, the RCT [9] showed a statistically significant reduction in the time taken to change dressings when SecurAcath was used [4.3 min (95\% CI 3.8-4.9) compared with 7.3 min (95\% CI 6.4-8.3) for StatLock $(p<0.0001)]$. This had an important bearing on the cost-effectiveness analysis (see below).

The EAC concluded that there was insufficient evidence to draw firm conclusions about the superiority of SecurAcath with regard to effectiveness and adverse events compared to
Table 1 Relative risks from Goossens et al. (2018) comparing SecurAcath to StatLock

\begin{tabular}{llll}
\hline Outcome & $\begin{array}{l}\text { SecurAcath results } \\
n / N(\%)\end{array}$ & $\begin{array}{l}\text { StatLock } \\
\text { results } \\
n / N(\%)\end{array}$ & $\begin{array}{l}\text { Individual relative risk } \\
\text { SecurAcath/StatLock } \\
(95 \% \mathrm{CI})\end{array}$ \\
\hline Migration & $3 / 51(5.9)$ & $2 / 51(3.9)$ & $1.50(0.31-7.35)$ \\
Dislodgement & $3 / 51(5.9)$ & $2 / 51(3.9)$ & $1.50(0.31-7.35)$ \\
Total catheter infection & $1 / 51(2.0)$ & $1 / 51(2.0)$ & $1.00(0.11-9.50)$ \\
CRBSI & $1 / 51(2.0)$ & $1 / 51(2.0)$ & $1.00(0.11-9.50)$ \\
\hline
\end{tabular}

$C I$ confidence interval, $C R B S I$ catheter-related blood stream infection

\begin{tabular}{|c|c|c|c|c|}
\hline Outcome & Intervention & No. of studies & Proportion $(\%)$ & $95 \% \mathrm{CI}$ \\
\hline \multirow[t]{3}{*}{ Migration } & SecurAcath & 3 & 4.0 & $1.5-8.5$ \\
\hline & StatLock & 4 & 4.7 & $2.3-8.5$ \\
\hline & Suture & 2 & 11 & $6.3-18.1$ \\
\hline \multirow[t]{3}{*}{ Dislodgement } & SecurAcath & 9 & 0.6 & $0.3-1.0$ \\
\hline & StatLock & 7 & 4.1 & $3.3-5.0$ \\
\hline & Suture & 3 & 8.7 & $4.8-14.2$ \\
\hline \multirow[t]{3}{*}{ Total catheter infections } & SecurAcath & 5 & 0.8 & $0.3-1.7$ \\
\hline & StatLock & 6 & 1.6 & $1.1-2.4$ \\
\hline & Suture & 3 & 6.8 & $3.5-11.95$ \\
\hline \multirow[t]{3}{*}{ CRBSI } & SecurAcath & 2 & 1.68 & $0.2-5.9$ \\
\hline & StatLock & 2 & 1.47 & $0.2-5.2$ \\
\hline & Suture & na & & \\
\hline \multirow[t]{3}{*}{ Unplanned removals } & SecurAcath & 3 & 15.53 & $10.3-22.6$ \\
\hline & StatLock & na & & \\
\hline & Suture & na & & \\
\hline \multirow[t]{3}{*}{ Unplanned reinsertions } & SecurAcath & 1 & 0 & $0-97.5^{\mathrm{a}}$ \\
\hline & StatLock & na & & \\
\hline & Suture & na & & \\
\hline
\end{tabular}

CI confidence interval, $C R B S I$ catheter-related blood stream infection, $n a$ not available

${ }^{\mathrm{a}} 95 \% \mathrm{CI}$ is one sided
Table 2 Pooled estimates from all studies included in the metaanalysis 
StatLock. The EAC concluded that there was some evidence that SecurAcath is non-inferior in effectiveness and sideeffect profiles to StatLock. The EAC noted that although the evidence suggests that both SecurAcath and StatLock are superior to sutures, this evidence is from a population requiring PICC lines, for which suturing is not relevant to clinical practice. Finally, the EAC concluded there is insufficient information to compare the effectiveness and safety of SecurAcath with its comparators in terms of the subgroups specified in the scope.

\subsection{Economic Evidence}

The EAC rejected the evidence included by the manufacturer as it was deemed out of scope because none of the studies focused on CVCs. The EAC ran a new comprehensive search based on the search strategy used for the clinician evidence. However, none of the studies found met the inclusion criteria defined in the scope.

The EAC questioned a number of the assumptions made in the manufacturer's de-novo cost model, which provided separate cost analyses for PICCs and CICCs. The sponsor's submission assumed a indwell time of 25 days for PICC lines and 3 days for CICC lines. The manufacturer's submission did not explicitly consider children or patients with comorbidities. The EAC regarded the separation of analyses for CICC and PICC lines to be appropriate. The EAC considered the assumptions on indwell times for catheters as conservative with respect to the cost analysis. Consequently, the addition of the sensitivity analysis considering medium or long indwell times would be very unlikely to change the inference arising from the analysis. The EAC considered it unlikely that sufficient data would be available to support a sensitivity analysis specifically addressing catheter securement in children or in patients with co-morbidities. The EAC accepted the possibility that younger age or comorbidities would influence the rate of CRBSI. The EAC accepted the possibility that youth or dementia would influence the rate of dislodgement by patients. The EAC regarded a sensitivity analysis on the parameters for the rate of CRBSI and the rate of dislodgement as sufficient to address concerns about the cost implications of the use of SecurAcath in children or patients with co-morbidities. The sensitivity analysis undertaken in the manufacturer's submission only partially addressed these concerns.

With regard to comparators, the manufacturer's model provided StatLock as the comparator for PICCs and sutures as the comparator for CICCs. The EAC decided that the manufacturer's submission had not fully addressed the specified scope in failing to include adhesive devices as a comparator to SecurAcath for the securement of CICC lines.

The manufacturer's model utilised a decision tree (see Fig. 1). The EAC regarded the sponsor's approach as reasonable with the caveat that the risk of complications would

SecurAcath model structure diagram

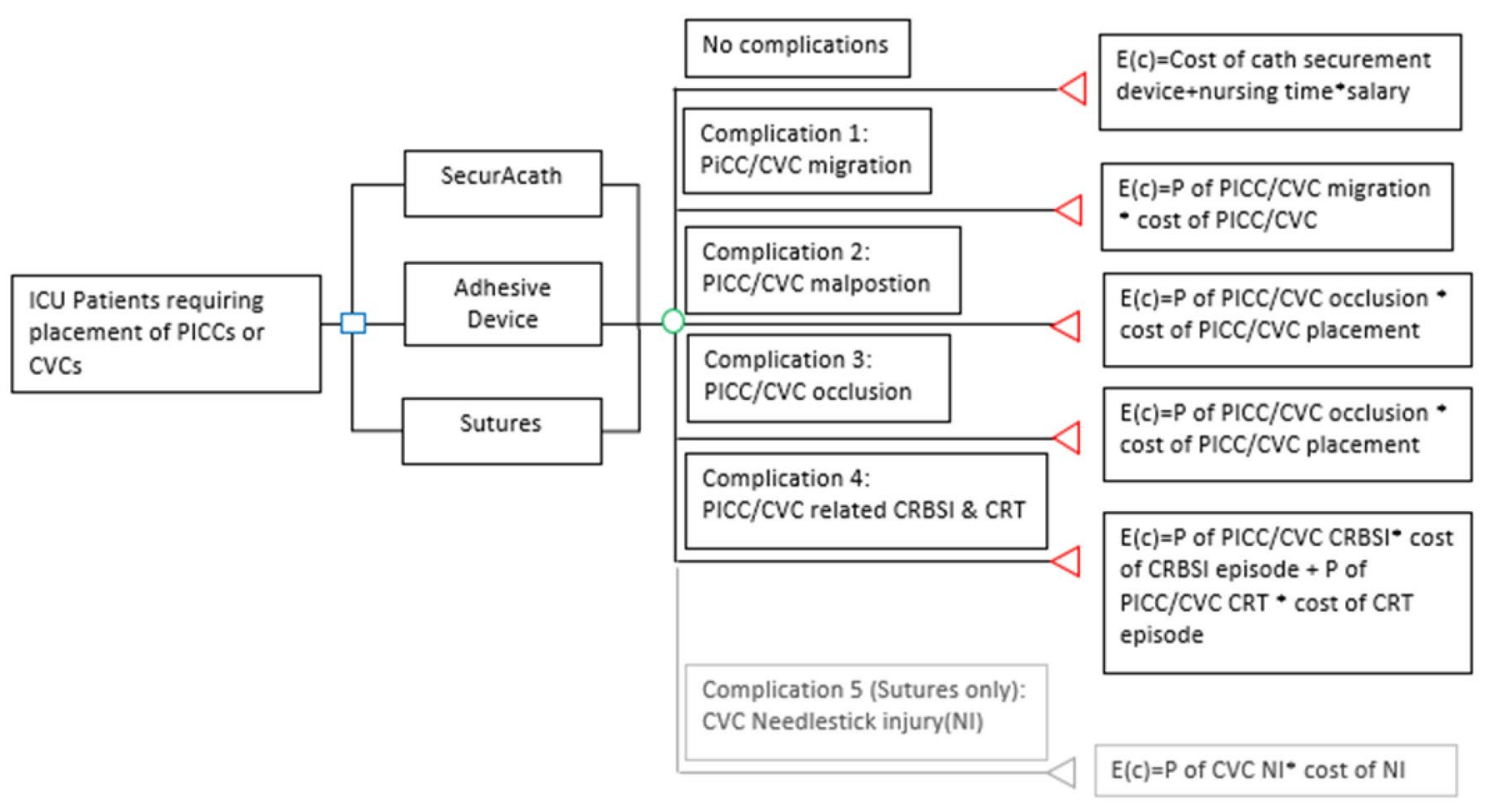

Fig. 1 SecurAcath model structure diagram. CRBSI catheter-related blood stream infection, $C R T$ catheter-related thrombosis, $C V C$ central venous catheter, $E(c)$ Expected cost, $I C U$ intensive care unit, $P$ prob- abilities, PICC peripherally inserted central catheter. The diagram is reproduced from the submission including apparent typographical errors 
be likely to vary with indwell time. The EAC regarded the structure of the decision trees as acceptable. The EAC considered the term 'malposition' to be vague in a clinical sense and that this term should be replaced with 'dislodgement'. The EAC agreed with the manufacturer that an assumption of short indwell times for catheters would generate a conservative estimate of any cost savings accruing from the securement with SecurAcath on the basis that device costs for SecurAcath were 'front-loaded' and with increased potential for cost savings over extended indwell periods. In summary, the EAC considered the model specification to be appropriate for the cost analysis.

The manufacturer considered five complications: migration of the catheter; malposition of the catheter; occlusion of the catheter line; CRBSI or catheter-related thrombosis; and (for suture securement only) risk of needle-stick injury to professionals. The values for parameters were predominantly sourced from a report of one Canadian hospital's experience of implementing SecurAcath along with anticoagulant impregnated catheters, plus other articles from the manufacturer's submission [9-12, 15, 23, 30, 31]. The EAC identified numerous flaws in the Canadian hospital report, highlighting potential risks of publication bias. The EAC concluded that the meta-analysis would be a better source for the probabilities. However, the EAC considered the manufacturer's choices on indwell times and their decision not to quantify the impact of securement devices on patient mortality or quality of life to be appropriate. The manufacturer's cost assumptions are outlined in Table 3.

For a PICC line, the manufacturer estimated a saving of $£ 41$ ( $£ 17$ under a worst-case scenario sensitivity analysis) with the use of SecurAcath instead of StatLock for an indwell time of 25 days. For a CICC line, the manufacturer estimated a saving of $£ 1006$ ( $£ 876$ under a worst-case scenario sensitivity analysis) with the use of SecurAcath instead of suturing for an indwell time of 3 days. The large cost savings in the analysis of CICC lines arose from a reduced risk of CRBSI with SecurAcath.

The EAC had three main concerns with the manufacturer's cost model: first, the manufacturer's event probabilities were constant with respect to indwell times. The EAC believed that an assumption of an exponential relationship with time was more appropriate. Second, the event probabilities were sourced from weak literature. The EAC believed that evidence from one RCT comparing SecurAcath and StatLock [9] and another RCT comparing StatLock and sutures [27] was more authoritative and reliable. Third, the manufacturer's assumption of the cost for nurse time ( $£ 0.60$ per minute) was a substantial underestimation; the EAC provided a more accurate figure of $£ 2.08$ per minute.

Based on evidence from the RCTs, the EAC re-estimated cost savings under an assumption of no difference in complication rates (CRBSI, migration, dislodgement) in the base case. The EAC did include a risk of needle-stick injury with sutures on the basis that an assumption of reduced risk with suture-less securement devices was highly likely. The EAC undertook a sensitivity analysis that included differential risks of migration, dislodgement and CRBSI. The EAC did not consider the risk of catheter-related thrombosis or occlusion on the grounds that there was insufficient evidence to determine the relative risk from different securement devices.

Table 3 Manufacturer and External Assessment Centre (EAC)-corrected cost assumptions

\begin{tabular}{|c|c|c|}
\hline Parameter & Manufacturer assumption & EAC assumption (if different) \\
\hline Cost of PICC securement & $£ 250[10]$ & $£ 274$ conventional blind placement [32] \\
\hline Cost of CICC placement & $£ 450$ by surgeon in operating theatre $[33,34]$ & $\begin{array}{l}£ 440 \text { after inflation ( } £ 150 \text { by nurse on ward, } £ 312 \\
\text { blind insertion, } £ 382 \text { image guided, by nurse) } \\
\text { [33] }\end{array}$ \\
\hline Minute of nurse time & $£ 0.60[35]$ & $£ 2.08[36]$ \\
\hline Minute of doctor time & $£ 1.47[35]$ & \\
\hline StatLock placement nurse time & $3 \min [35]$ & \\
\hline SecurAcath placement nurse time & $0.5 \min [35]$ & \\
\hline Suturing doctor time & $4.7 \min [35]$ & In the UK, suturing would be done by a nurse \\
\hline Migration & $£ 250$ & $£ 134[33]$ \\
\hline CRBSI episode & $£ 9900[37]$ & (EAC noted a wide range $£ 812-71,000[38]$ ) \\
\hline Catheter-related thrombosis & $£ 250[37]$ & The figure is actually for local site infections \\
\hline Cost per needle-stick injury & $£ 312$ [39] & (EAC noted a wide range $£ 48-1516$ [40]) \\
\hline List price SecurAcath & $£ 16$ & \\
\hline Adhesive device & $£ 12$ for 4 devices in 25 -d indwell time & \\
\hline Sutures & $£ 5$ & \\
\hline
\end{tabular}

$C I C C$ centrally inserted central catheter, $C R B S I$ catheter-related blood stream infection, $P I C C$ peripherally inserted central catheter 
For CICC lines, the EAC compared SecurAcath with both StatLock and sutures, while for PICC lines SecurAcath was compared with StatLock only. Three indwell times were considered for both CICC and PICC lines: 5 days (short); 25 days (medium); and 120 days (long). In the base-case, cost estimates were based on placement and maintenance costs over the relevant indwell time with the inclusion of costs related to the risk of needle-stick injury at the placement of the catheter where relevant. The sensitivity analysis included costs attributable to migration, dislodgement and CRBSI. These costs were estimated as the product of the probability of the event over the indwell period and the unit cost of the event. The EAC assumed that complication rates were independent of whether the catheter was a PICC or CICC.
The EAC assumed a placement time for StatLock of 3 min [31] and, owing to a lack of evidence, the same was assumed for SecurAcath. (The manufacturer assumed a placement time of $30 \mathrm{~s}$ and this was addressed in the EAC's sensitivity analyses.) Dressing change times (4.3 min for SecurAcath, 7.3 min for StatLock) and needle-stick injury risks $(1.2 \%$ per procedure) were taken from the RCTs [9, 27]. The parameters used in the EAC's model are listed in Table 4.

\subsubsection{Results}

In the base-case analysis (see Table 5), the cheapest option was StatLock for short indwell times (PICC and CICC), SecurAcath for PICC (medium and long indwell times) and
Table 4 Parameters used in the External Assessment Centre cost model

\begin{tabular}{|c|c|c|}
\hline Parameter & Value (base case) & $\begin{array}{l}\text { Value } \\
\text { (sensitivity } \\
\text { analysis) }\end{array}$ \\
\hline \multicolumn{3}{|c|}{ Routine placement and maintenance times } \\
\hline SecurAcath placement & $3 \min$ & $0.5 \min$ \\
\hline StatLock placement & $3 \min$ & \\
\hline Suture placement & $4.7 \mathrm{~min}$ & \\
\hline SecurAcath maintenance & $4.3 \mathrm{~min}$ & \\
\hline StatLock maintenance & $7.3 \mathrm{~min}$ & \\
\hline Suture maintenance & $4.3 \mathrm{~min}$ & $7.3 \mathrm{~min}$ \\
\hline \multicolumn{3}{|c|}{ Hazard ratios (SecurAcath vs. StatLock) } \\
\hline Migration & & 0.8443 \\
\hline Dislodgement & & 0.1424 \\
\hline CRBSI & & 1.1441 \\
\hline \multicolumn{3}{|c|}{ Complication rates per 1000 catheter days } \\
\hline SecurAcath migration & 0 & 1.52 \\
\hline StatLock migration & 0 & 1.8 \\
\hline Suture migration & 0 & 3.1 \\
\hline SecurAcath dislodgement & 0 & 0.51 \\
\hline StatLock dislodgement & 0 & 3.6 \\
\hline Suture dislodgement & 0 & 4.1 \\
\hline SecurAcath CRBSI & 0 & 0.80 \\
\hline StatLock CRBSI & 0 & 0.7 \\
\hline Suture CRBSI & 0 & 3.4 \\
\hline Needle-stick injury (suture) ${ }^{\mathrm{a}}$ & 1.2 & \\
\hline \multicolumn{3}{|l|}{ Unit costs } \\
\hline Nurse time per minute & $£ 2.08$ & \\
\hline Cost of CRBSI & $£ 9900$ & \\
\hline Cost of needle-stick Injury & $£ 312$ & \\
\hline Cost of migration of CICC line & $£ 134$ & \\
\hline Cost of migration of PICC line & $£ 134$ & \\
\hline Cost of dislodgement of CICC line & $£ 440$ & \\
\hline Cost of dislodgement of PICC line & $£ 274$ & \\
\hline
\end{tabular}

$C I C C$ centrally inserted central catheter, $C R B S I$ catheter-related blood stream infection, PICC peripherally inserted central catheter

${ }^{\mathrm{a}}$ Rate per 1000 procedures 
Table 5 External Assessment Centre's base-case analysis

\begin{tabular}{|c|c|c|c|c|c|}
\hline Scenario & SecurAcath $(£)$ & StatLock $(£)$ & Sutures $(£)$ & Cheapest option & Saving (£) \\
\hline CICC line for $5 \mathrm{~d}$ & 22 & 10 & 15 & StatLock & 5 \\
\hline PICC line for $5 \mathrm{~d}$ & 22 & 10 & - & StatLock & 12 \\
\hline CICC line for $25 \mathrm{~d}$ & 49 & 66 & 42 & Sutures & 7 \\
\hline PICC line for $25 \mathrm{~d}$ & 49 & 66 & - & SecurAcath & 17 \\
\hline CICC line for $120 \mathrm{~d}$ & 174 & 268 & 167 & Sutures & 7 \\
\hline PICC line for $120 \mathrm{~d}$ & 174 & 268 & - & SecurAcath & 94 \\
\hline
\end{tabular}

CICC centrally inserted central catheter, PICC peripherally inserted central catheter sutures for CICC (medium and long indwell times). Oneway sensitivity analysis (using the manufacturer's assumption of a 30 -second placement time) resulted in a $£ 5$ saving for SecurAcath but did not alter the results. However, assuming a suture maintenance time of $7.3 \mathrm{~min}$ (equivalent to StatLock) resulted in SecurAcath becoming the cheapest option for both PICC and CICC over medium and long indwell times. The multi-way sensitivity analysis, in which differences in adverse events were assumed, generated a similar inference with regard to the relative costs of SecurAcath and Statlock, but costs for suturing rose substantially (see Table 6).

The threshold sensitivity analysis for the best-case scenario indicated that the costs of SecurAcath dropped below those of StatLock for securing PICC lines at indwell times of 15 days or more. The costs of sutures dropped below those of StatLock for securing CICC lines at indwell times of 8 days or more. SecurAcath remained more expensive than sutures for securing CICC lines over any indwell time. The probabilistic sensitivity analysis generated mean cost savings of $£ 22$ (95\% CI $-£ 128$ to $£ 438$ ) for securing PICC lines with SecurAcath, compared to StatLock, for an indwell time of 25 days. For a CICC line with an indwell time of 5 days, the probabilistic sensitivity analysis generated mean cost savings of $-£ 7$ (95\% CI $-£ 210$ to $£ 47$ ) for SecurAcath, compared to StatLock, and $£ 137$ (95\% CI - £31 to $£ 574$ ) when compared to suturing.

The EAC concluded that although there is considerable uncertainty, reflected in the large confidence intervals in the meta-analysis, SecurAcath appears to be cheaper than StatLock over medium and long indwell times. This is because of the savings on maintenance costs arising from a reduction in cleaning time [9] and the need to replace StatLock on a weekly basis. StatLock was cheaper than SecurAcath in all scenarios for short indwell times. The EAC noted that although there is some evidence that StatLock has an increased risk of adverse events, the absolute risk is likely to be small over an indwell time of 5 days.

The EAC found the greatest uncertainties in comparing SecurAcath with sutures. In the base-case analysis, sutures were shown to be slightly cheaper. However, when the CRBSI risk was taken into account for the multi-way sensitivity analysis, SecurAcath was found to be cheaper.

\section{Conclusions of the EAC}

The EAC critiqued the manufacturer's submission and made substantial revisions to their choice of included studies. The EAC ran a new search and undertook a meta-analysis. The meta-analysis included low-quality observational and noncomparative evidence, as well as evidence from RCTs that did not include SecurAcath [22, 25, 27]. However, the findings were broadly in line with the strongest available evidence (one RCT comparing SecurAcath and StatLock [9]) and the EAC found that SecurAcath and StatLock are superior to sutures for migration, dislodgement, total catheterrelated infections and CRBSIs. However, it should be noted that this evidence relates to people requiring PICC lines, for which sutures are not currently standard practice in the UK. The EAC concluded that there is insufficient evidence to determine that SecurAcath is clinically superior to StatLock
Table 6 External Assessment Centre's multi-way sensitivity analysis

\begin{tabular}{|c|c|c|c|c|c|}
\hline Scenario & SecurAcath (f) & StatLock (f) & Sutures $(\mathfrak{f})$ & Cheapest option & Saving $(\mathfrak{f})$ \\
\hline CICC line for $5 \mathrm{~d}$ & 64 & 53 & 193 & StatLock & 11 \\
\hline PICC line for $5 \mathrm{~d}$ & 64 & 50 & - & StatLock & 14 \\
\hline CICC line for $25 \mathrm{~d}$ & 256 & 281 & 902 & SecurAcath & 25 \\
\hline PICC line for $25 \mathrm{~d}$ & 254 & 267 & - & SecurAcath & 13 \\
\hline CICC line for $120 \mathrm{~d}$ & 1130 & 1246 & 3696 & SecurAcath & 116 \\
\hline PICC line for $120 \mathrm{~d}$ & 1120 & 1188 & - & SecurAcath & 68 \\
\hline
\end{tabular}

$C I C C$ centrally inserted central catheter, $P I C C$ peripherally inserted central catheter 
but there is some evidence that SecurAcath is non-inferior to StatLock in effectiveness and side-effect profiles.

The EAC made a number of revisions to the manufacturer's cost model, relating to a variety of the assumptions made on costs and the risk of adverse events. Contrary to the manufacturer's analysis, in all scenarios, StatLock was shown to be the cheapest option for short indwell times. The base-case analysis showed SecurAcath to be the cheaper option for medium and long indwell times for PICC lines only. However, consideration of potential differences in CRBSI rates led to sharply higher costs for suturing. Therefore, the EAC concludes that it is likely that SecurAcath is associated with the lowest costs for securement of PICC or CICC lines over medium and long indwell times. The savings are driven by a reduced need for replacement devices as well as significantly shorter maintenance times.

\section{NICE Guidance}

In line with the Medical Technologies Evaluation Programme process, the MTAC met to develop draft recommendations following which a medical technology consultation document was produced. Comments were accepted by NICE on these draft recommendations as well as the notification of inaccuracies and additional information. Following a consultation period, comments were collated and presented to the MTAC for discussion.

\subsection{Draft Recommendations}

The MTAC met in December 2016 and, following a review of the manufacturer's submissions and the EAC report, [41] together with evidence from expert advisers, the following provisional recommendations were made:

1. The case for adopting SecurAcath for securing PICCs is supported by the evidence. SecurAcath is easy to insert, well tolerated, associated with a low incidence of catheter-related complications and does not usually need replacing while the catheter is in place.

2. SecurAcath should be considered for any PICC inserted for an anticipated medium- to long-term indwell time (15 days or more).

3. Cost modelling shows that SecurAcath leads to cost savings if the PICC remains in place for 15 days or longer. Estimated cost savings range from $£ 13$ to $£ 99$ per patient for indwell times of 25 days and 120 days, respectively. Cost savings result from shorter maintenance times and a reduced need for device replacement with SecurAcath when compared with adhesive securement devices. Annual savings across the NHS from using SecurAcath are estimated to be around $£ 2$ million.

\subsection{Consultation Response}

During the public consultation period, NICE received comments from interested stakeholders, including the manufacturer, the Department of Health, professional societies and private sector practitioners. The committee reviewed the comments and decided to reword certain sections of the guidance document, as well as updating the cost model after receiving more precise prices from the manufacturer. During this period, one of the conference abstracts referenced by the EAC was published as a full paper [21] but the reported outcomes did not materially affect the draft recommendations.

\subsection{Final Guidance}

The MTAC considered the results of the consultation, and the final Medical Technology Guidance document for SecurAcath for securing percutaneous catheters was published by NICE in June 2017 [42]. There were minor changes to the provisional recommendations highlighted in bold below.

1. The case for adopting SecurAcath for securing PICCs is supported by the evidence. SecurAcath is easy to insert, well tolerated, associated with a low incidence of catheter-related complications and does not usually need removing while the catheter is in place.

2. SecurAcath should be considered for any PICC with an anticipated medium- to long-term indwell time (15 days or more).

3. Cost modelling shows that SecurAcath is cost saving compared with adhesive securement devices if the PICC remains in place for 15 days or longer. Estimated cost savings range from $£ 9$ to $£ 95$ per patient for indwell times of 25 days and 120 days, respectively. Cost savings result from shorter maintenance times and less need for device replacement with SecurAcath. Annual savings across the NHS in England from using SecurAcath are estimated to be a minimum of $£ 4.2$ million.

\section{Challenges}

The EAC encountered a number of challenges during the composition of its report, chiefly relating to a problem common to reviews for the Medical Technologies Evaluation Programme: a lack of comparative evidence for the device in question. Only one of the studies [9] included in this report actually compared SecurAcath with another type of catheter securement, StatLock. At the time the EAC was compiling the assessment report, this single RCT was only available as a pre-publication draft, obtained following a request to the authors. Only one other study reporting on SecurAcath [12] had adequate 
baseline characteristics of the study populations, including sex and age, vein used and indication for PICC insertion. Consequently, it was impossible to perform any sub-group analyses. Furthermore, it was impossible to evaluate the manufacturer's view that SecurAcath is likely to be predominantly used in older critically ill patients, who are likely to have a number of co-morbidities and in patients following major trauma, or those with conditions requiring long-term ongoing therapy such as cancer.

The meta-analysis undertaken by the EAC provided a more reliable grounding on which much of the report was based, including the cost-effectiveness analyses. However, there were numerous challenges in carrying out the metaanalysis owing to the paucity of comparable data. The majority of the evidence comprised conference abstracts or poster presentations, which lacked demographic information, thus it was impossible to assess the heterogeneity of the studies. The EAC's approach was to pool the available data for five outcomes (migration, dislodgement, catheterrelated infection, CRBSI, unplanned removals/reinsertions) and to calculate relative risks for each of these. The EAC also included studies that did not include SecurAcath $[22,25,27]$ but provided data on StatLock and sutures. The EAC concluded that despite these limitations there was value in conducting a meta-analysis that provided at least an indication of the difference in clinical outcomes between SecurAcath and its comparators.

The EAC concluded that there was insufficient evidence to show SecurAcath was clinically superior to its comparators and although the EAC's cost model showed, in some scenarios, that SecurAcath was the cheapest option, there were numerous caveats surrounding the quality of the available evidence for many of the assumptions. The MTAC looked beyond the EAC's report to the experiences of clinicians who had used the device: ten nurses and other specialists provided responses to a questionnaire [43]. There was an overwhelmingly positive assessment of SecurAcath from the clinicians using the device in practice. The most common benefit reported by clinicians was the reduced risk of migration, reduced number of $\mathrm{x}$-rays for reinsertion and the improved ease of cleaning the catheter exit site. Another commonly voiced benefit was that SecurAcath provided patients with increased confidence when moving their arms. A selection of the comments is presented below.

"I realised that we were able to cleanse the exit site more effectively without the fear of pulling the line out."

"Given there is no adhesive required it has a major advantage over competitors and allows cleansing of the skin to be undertaken between dressing changes."
"Its unique design means it provides securement of a $\mathrm{CVC}$ and does not require changing has resulted in a huge reduction malpositioned PICCs."

"Previous securement methods have involved the use of adhesive plasters which are placed on the skin. In our experience these were unreliable and lead to many instances of catheter migration."

"There are various other devices for securing PICCs but unlike SecurAcath they are adhesive devices which have to be changed once a week. Changing the dressing and the device carries a high risk of dislodgement."

"There is no doubt in my mind that this product has produced significant benefits to our patients in terms of the outcome measures."

"There are costs involved with regularly changing PICC adhesive securement devices-these would be reduced by using this technology."

"In situations where it replaces Statlock, the cost of a SecurAcath and tissue adhesive will quickly be saved within a few weeks for a given patients because Statlock has to be replaced weekly."

There were a small number of negative comments about the device, chiefly on the issue of pain at removal.

"Removal of the device can cause pain in about half of patients. Local anaesthetic can be used if they experience discomfort when the device is wiggled but local anaesthetic itself causes brief pain when administered."

"Some patients may have an allergy to nickel—not many in our experience."

"The success of this device relies on proper training of those inserting and removing it and those providing line care ... Another potential obstacle is that removing the device can be daunting to those with no experience."

The decision by MTAC to recommend SecurAcath was taken on the basis of the available clinical evidence (pointing to non-inferiority), a cost-effectiveness model (cost saving in a number of scenarios) and the expert opinions of clinicians using the device (overwhelmingly positive).

\section{Conclusion}

Despite a challenging evidence base, the EAC was able to deliver a cogent assessment report and this, combined with expert clinical opinion, was used by the MTAC to make a 
positive recommendation for SecurAcath to be used in the NHS.

Author Contributions AC, JAS, JLP, KG, TM and NH contributed to the clinical effectiveness section. MP and DZ contributed to the economics section. The manuscript was prepared by TM. The guarantors for overall content are SK and AC. This summary has not been externally peer reviewed by Applied Health Economics and Health Policy.

\section{Compliance with Ethical Standards}

Funding This work was supported by the Wellcome/Engineering and Physical Sciences Research Council Centre for Medical Engineering (WT 203148/Z/16/Z), the National Institute for Health and Care Excellence, and the National Institute for Health Research Biomedical Research Centre based at Guy's and St Thomas' NHS Foundation Trust and King's College London. The views expressed are those of the author(s) and not necessarily those of the National Health Service, the National Institute for Health Research, or the Department of Health and Social Care.

Conflict of interest Janet L. Peacock is a National Institute for Health Research Senior Investigator. Tom Macmillan, Mark Pennington, Jennifer A. Summers, Kate Goddard, Darshan Zala, Naomi Herz, Stephen Keevil and Anastasia Chalkidou have no conflicts of interest that are directly relevant to the contents of this article.

Open Access This article is distributed under the terms of the Creative Commons Attribution-NonCommercial 4.0 International License (http://creativecommons.org/licenses/by-nc/4.0/), which permits any noncommercial use, distribution, and reproduction in any medium, provided you give appropriate credit to the original author(s) and the source, provide a link to the Creative Commons license, and indicate if changes were made.

\section{References}

1. NICE. Medical technologies evaluation programme methods guide: process and methods [PMG33]. NICE; 2017. https://www. nice.org.uk/process/pmg33/.

2. NICE. Medical technologies evaluation programme process guide: process and methods [PMG34]. NICE; 2017. https://www.nice. org.uk/process/pmg34/.

3. Radhakrishnan M, Peacock J, Rua T, Clough RE, Ofuya M, Wang $\mathrm{Y}$, et al. E-vita open plus for treating complex aneurysms and dissections of the thoracic aorta: a NICE Medical Technology Guidance. Appl Health Econ Health Policy. 2014;12(5):485-95. https://doi.org/10.1007/s40258-014-0114-9.

4. Gorski, LA. The 2016 infusion therapy standards of practice. Home Healthcare Now. 2017;35(1):10-8.

5. Royal Marsden Manual of Clinical Nursing Procedures. 9th ed. Chichester: Royal Marsden NHS Foundation Trust; 2015.

6. Smith RN, Nolan JP. Central venous catheters. BMJ. 2013;347.

7. Office NA. Progress in improving cancer services and outcomes in England. 2015. Available from: https://www.nao.org.uk/report/ progress-in-improving-cancer-services-and-outcomes/. Accessed 14 Aug 2018.

8. Jabeen S, Murtaza G, Hanif MZ, Morabito A, Khalil B. Migration of indwelling central venous catheter and fatal hydrothorax. Eur J Pediatr Surg Rep. 2014;2(1):32-4. https://doi. org/10.1055/s-0033-1347130.

9. Goossens GA, Grumiaux N, Janssens C, Jerome M, Fieuws S, Moons P, et al. SecurAstaP trial: securement with SecurAcath versus StatLock for peripherally inserted central catheters, a randomised open trial. BMJ Open. 2018;8(2):e016058. https://doi. org/10.1136/bmjopen-2017-016058.

10. Hughes EM. Reducing PICC migrations and improving patient outcomes. Br J Nurs. 2014;23(2):S12, S4-8.

11. Cordovani D, Cooper RM. A prospective trial on a new sutureless securement device for central venous catheters. Can J Anaesth. 2013;60(5):504-5. https://doi.org/10.1007/s12630-013-9897-7.

12. Egan GM, Siskin GP, Weinmann R, Galloway MM. A prospective postmarket study to evaluate the safety and efficacy of a new peripherally inserted central catheter stabilization system. J Infus Nurs. 2013;36(3):181-8. https://doi.org/10.1097/ NAN.0b013e3182893690.

13. Ballance P. Evaluation of products promote patient and clinician with positive advancement in vascular access care. Poster presentation. 2012.

14. Djurcic-Jovan A, Eisener A, McDiarmid S. Implementation of a quality improvement initiative reduces PICC migrations in a complex continuing care hospital. Poster presentation for Canadian Vascular Access Association. 2016.

15. Anonymous. Misericordia parenteral therapy PICC statistics/ outcomes report. Report from Covenant Health, Canada. 2015.

16. McParlan D, Gault M, Gillespie R. PICC migration: a problem of the past! Poster presentation. 2016.

17. Pittiruti M. Defining the indications of subcutaneously anchored securement devices: a European point of view. Poster presentation. 2015 .

18. Sandeluss S, Price N, Simcock L. Introducing SecurAcath into a haematology/oncology setting. Poster presentation. 2013.

19. Stone L, Lamagna P, Pratt P. Improving PICC care in the pediatric patient. Poster presentation. 2013.

20. Hill JG. Beyond the dressing for PICCs: strategy for cost-savings and work efficiency. J Vasc Access. 2014;15(3):208. https ://doi.org/10.5301/jva.5000275.

21. Zerla PA, Canelli A, Cerne L, Caravella G, Gilardini A, De Luca G, et al. Evaluating safety, efficacy, and cost-effectiveness of PICC securement by subcutaneously anchored stabilization device. J Vasc Access. 2017;18(3):238-42. https://doi. org/10.5301/jva.5000655.

22. Fang F, Zhang HY, Wang F, Yang W, Zhang JL. Comparison of three different fixiation methods for peripherally inserted central catheter [in Chinese]. Chin J Clin Nutr. 2011;19(2):119-23. https://doi.org/10.3760/cma.j.issn.1674-635X.2011.02.013.

23. McMahon DD. Evaluating new technology to improve patient outcomes: a quality improvement approach. J Infus Nurs. 2002;25(4):250-5.

24. Sansivero G, Siskin G, Tessier M, MacDowell B. Securacath subcutaneous securement in peripherally inserted central catheters: results of a prospective 50 patient trial with an internal securement device. J Vasc Access. 2011;12(1):81. https://doi. org/10.5301/jva.2011.6368

25. Teichgraber UK, de Bucourt M, Gebauer B, Streitparth F, Hamm B, Enzweiler C. Effectiveness of sutureless percutaneous placement of cuffed tunneled hemodialysis catheters applying StatLock attachment devices. J Vasc Access. 2011;12(1):17-20.

26. Venturini M, Zaccomer F, Nadbath P, Bracca A, Nin C, Paroni R, et al. Efectiveness of securing central venous catheters in hematologic patients with sutureless percutaneous catheter attachment devices. Haematologica. 2011;96:338.

27. Yamamoto AJ, Solomon JA, Soulen MC, Tang J, Parkinson K, Lin R, et al. Sutureless securement device reduces complications 
of peripherally inserted central venous catheters. J Vasc Interv Radiol. 2002;13(1):77-81.

28. Zerla PA, Canelli A, Caravella G, Gilardini A, De Luca G, Parini $\mathrm{R}$, et al. Open- vs closed-tip valved peripherally inserted central catheters and midlines: findings from a vascular access database. J Assoc Vasc Access. 2015;20(3):169-76. https://doi.org/10.1016/j. java.2015.06.001.

29. Miettinen O, Nurminen M. Comparative analysis of two rates. Stat Med. 1985;4(2):213-26. https://doi.org/10.1002/sim.4780040211.

30. Ahmed Z, Mohyuddin Z. Complications associated with different insertion techniques for Hickman catheters. Postgrad Med J. 1998;74(868):104-7.

31. Frey AM, Schears GJ. Why are we stuck on tape and suture? A review of catheter securement devices. J Infus Nurs. 2006;29(1):34-8.

32. NICE. The Sherlock 3CG Tip confirmation system for placement of peripherally inserted central catheters. Medical Technologies Guidance. MTG24; Mar 2015.

33. Boland A, Haycox A, Bagust A, Fitzsimmons L. A randomised controlled trial to evaluate the clinical and cost-effectiveness of Hickman line insertions in adult cancer patients by nurses. Health Technol Assess. 2003;7(36):112. https://doi.org/10.3310/hta7360.

34. Hamilton H. Care improves while costs reduce: the clinical nurse specialist in total parenteral nutrition. Prof Nurse. 1993;8(9):592-4,6.

35. Medical I. SecurAcath cost-effectiveness report. Plymouth, Minnesota: Interrad Medical; 2015.

36. Curtis L, Burns A. Unit costs of health and social care 2015, personal social servicesresearch unit. Canterbury: University of Kent; 2015.
37. NICE. The $3 \mathrm{M}$ Tegaderm CHG IV securement dressing for central venous and arterial catheter insertion sites. Medical Technologies Guidance. MTG25. NICE; 2015. https://www.nice.org.uk/ guidance/mtg25.

38. Hockenhull JC, Dwan K, Boland A, Smith G, Bagust A, Dündar $\mathrm{Y}$, et al. The clinical effectiveness and cost-effectiveness of central venous catheters treated with anti-infective agents in preventing bloodstream infections: a systematic review and economic evaluation. Health Technol Assess. 2008;12(12):154. https://doi. org/10.3310/hta12120.

39. Lee WC, Nicklasson L, Cobden D, Chen E, Conway D, Pashos CL. Short-term economic impact associated with occupational needlestick injuries among acute care nurses. Curr Med Res Opin. 2005;21(12):1915-22. https://doi.org/10.1185/030079905x65286.

40. Mannocci A, De Carli G, Di Bari V, Saulle R, Unim B, Nicolotti $\mathrm{N}$, et al. How much do needlestick injuries cost? A systematic review of the economic evaluations of needlestick and sharps injuries among healthcare personnel. Infect Control Hosp Epidemiol. 2016;37(6):635-46. https://doi.org/10.1017/ice.2016.48.

41. Chalkidou A, Goddard K, Herz N, Keevil S, Macmillan T, Peacock J, et al. External Assessment Centre report: the SecurAcath device for securing percutaneous catheters. NICE; 2016. https:// www.nice.org.uk/guidance/mtg34/documents/assessment-report.

42. NICE. SecurAcath for securing percutaneous catheters. Medical Technologies Guidance. MTG34. NICE; 2017. https://www.nice. org.uk/guidance/mtg34.

43. NICE. EAQ collated responses. 2017. https://www.nice.org.uk/ guidance/mtg34/documents/eaq-collated-responses. Accessed 14 Aug 2018. 